\title{
Gene expression profiling with microarray and SAGE identifies PLUNC as a marker for hepatoid adenocarcinoma of the stomach
}

\author{
Kazuhiro Sentani ${ }^{1}$, Naohide Oue ${ }^{1}$, Naoya Sakamoto ${ }^{1}$, Koji Arihiro ${ }^{2}, K_{\text {Kazuhiko Aoyagi }}^{3}$, \\ Hiroki Sasaki ${ }^{3}$ and Wataru Yasui ${ }^{1}$ \\ ${ }^{1}$ Department of Molecular Pathology, Hiroshima University Graduate School of Biomedical Sciences, \\ Hiroshima, Japan; ${ }^{2}$ Department of Anatomical Pathology, Hiroshima University Hospital, Hiroshima, Japan \\ and ${ }^{3}$ Genetics Division, National Cancer Center Research Institute, Tokyo, Japan
}

\begin{abstract}
Gastric cancer is one of the most common malignancies worldwide. In this study, we screened for genes upregulated in gastric cancer by comparing gene expression profiles from serial analysis of gene expression and microarray and identified the palate, lung, and nasal epithelium carcinoma-associated protein (PLUNC) gene. Immunostaining for PLUNC in $\mathbf{1 4 0}$ gastric cancer cases revealed strong and extensive staining of PLUNC in hepatoid adenocarcinoma of the stomach, whereas $7 \%$ of conventional gastric cancer cases showed focal immunostaining of PLUNC. Gastric hepatoid adenocarcinoma is an extrahepatic tumor characterized by morphologic similarities to hepatocellular carcinoma. To investigate the utility of PLUNC immunostaining in the diagnosis of gastric hepatoid adenocarcinoma, six cases of gastric hepatoid adenocarcinoma (six primary tumors and two associated liver metastases) were studied further. PLUNC staining was observed in all six primary hepatoid adenocarcinomas. PLUNC staining was observed in both the hepatoid adenocarcinoma and tubular/papillary adenocarcinoma components of primary tumors, although PLUNC staining was preferentially localized in tubular/papillary adenocarcinoma components. Staining of PLUNC was also detected in both liver metastases. PLUNC staining was not observed in $\mathbf{5 2}$ cases of primary hepatocellular carcinoma or in normal adult or fetal liver. These results indicate that PLUNC is a novel marker that distinguishes gastric hepatoid adenocarcinoma from primary hepatocellular carcinoma.
\end{abstract}

Modern Pathology (2008) 21, 464-475; doi:10.1038/modpathol.3801050; published online 18 January 2008

Keywords: hepatoid adenocarcinoma; hepatocellular carcinoma; PLUNC; microarray; SAGE; gastric cancer

According to the World Health Organization, gastric cancer is the fourth most common malignancy worldwide, with approximately 870000 new cases occurring every year. Mortality due to gastric cancer is second only to that due to lung cancer. ${ }^{1}$ Cancer develops as a result of multiple genetic and epigenetic alterations. ${ }^{2-4}$ Better knowledge of the changes in gene expression that occur during gastric carcinogenesis may lead to improvements in diagnosis, treatment, and prevention. Identification of novel biomarkers for cancer diagnosis and novel targets for treatment are the major goals in this field. ${ }^{5}$ To identify potential molecular markers for

Correspondence: Dr W Yasui, MD, PhD, Department of Molecular Pathology, Hiroshima University Graduate School of Biomedical Sciences, 1-2-3 Kasumi, Minami-ku, Hiroshima 734-8551, Japan. E-mail: wyasui@hiroshima-u.ac.jp

Received 29 June 2007; revised 10 September 2007; accepted 14 December 2007; published online 18 January 2008 cancer and to better understand the development of cancer at the molecular level, comprehensive gene expression analysis may be useful. Among the comprehensive methods used to analyze transcript expression levels, array-based hybridization ${ }^{6}$ and serial analysis of gene expression $(\mathrm{SAGE})^{7}$ are currently the most common approaches.

We previously performed SAGE of four primary gastric cancers. ${ }^{8}$ From the SAGE data, we identified several gastric cancer-associated genes; ${ }^{9}$ however, these alterations cannot completely explain the pathogenesis of gastric cancer. In addition, although gene expression profiles from SAGE and microarray have better correlations for genes with high-fold changes, the gene expression profiles from these methods show relatively poor correlations among genes with low-fold changes, suggesting that SAGE data may not yield a comprehensive gene expression profile. ${ }^{10}$ In our previous study, the invasion/ metastasis-associated genes identified by SAGE 
were quite different from those identified by microarray. ${ }^{11}$ Therefore, we performed gene expression profiling using Affymetrix GeneChip Human Genome U133Plus 2.0 arrays of one gastric cancer sample previously analyzed by SAGE and identified several candidate gastric cancer-associated genes. Among these candidate genes, the palate, lung, and nasal epithelium carcinoma-associated protein (PLUNC, also known as lung-specific X protein, $L U N X)$ gene is upregulated in human gastric cancer. However, little is known about the relation of PLUNC to human gastric cancer.

PLUNC was originally identified in the nasal epithelium of mouse embryo and the trachea and bronchi of adult mouse lung. ${ }^{12}$ The human PLUNC gene shows a similar expression pattern, including localization to the tracheal epithelium. ${ }^{13}$ PLUNC was also identified as a marker of non-small-cell lung carcinoma. $^{14}$ Immunohistochemical analysis of PLUNC in lung cancer revealed that PLUNC is commonly expressed in adenocarcinoma, mucoepidermoid carcinoma, and bronchoalveolar carcinoma and is absent from small cell carcinoma and squamous cell carcinoma. ${ }^{15}$ Although PLUNC is a major secreted protein product in the upper respiratory tract, ${ }^{15}$ the biologic function of PLUNC is poorly understood.

In this study, we examined the expression and distribution of PLUNC in human gastric cancer by immunohistochemistry. The relation between staining for PLUNC and clinicopathologic characteristics was examined. In addition, because we observed frequent immunostaining of PLUNC in hepatoid adenocarcinoma of the stomach, we also performed PLUNC immunostaining of primary hepatocellular carcinoma to investigate the potential utility of PLUNC immunostaining in the diagnosis of gastric hepatoid adenocarcinoma.

\section{Materials and methods}

\section{Tissue Samples}

In total, specimens from 144 cases of primary gastric cancer and 52 cases of primary hepatocellular carcinoma were collected. Patients were treated at the Hiroshima University Hospital. The histologic classification was based on the World Health Organization system. Tumor staging was according to the TNM classification system. ${ }^{16}$ Because written informed consent was not obtained, for strict privacy protection, identifying information for all samples was removed before analysis. This procedure was in accordance with the Ethical Guidelines for Human Genome/Gene Research of the Japanese Government. For microarray analysis, one primary gastric cancer sample (Case P208T, 60-year-old man, T4N3M0, stage IV, poorly differentiated adenocarcinoma) and corresponding non-neoplastic mucosa were used. This gastric cancer sample was analyzed previously by SAGE for comprehensive gene expression profiling. ${ }^{8}$ For quantitative reverse transcription
(RT)-polymerase chain reaction (PCR) analysis, four gastric cancer samples and corresponding nonneoplastic mucosa samples were used. The samples were obtained during surgery at the Hiroshima University Hospital. We confirmed microscopically that the tumor specimens were predominantly ( $>50 \%$ ) cancer tissue. Samples were frozen immediately in liquid nitrogen and stored at $-80^{\circ} \mathrm{C}$ until use. Samples of normal brain, spinal cord, heart, skeletal muscle, lung, stomach, small intestine, colon, liver, pancreas, kidney, bone marrow, spleen, peripheral leukocytes, and trachea were purchased from Clontech (Palo Alto, CA, USA). For western blot analysis, lysates from normal adult stomach and trachea were purchased from Clontech. For immunohistochemical analysis, we used archival formalin-fixed, paraffin-embedded tissues from 144 patients who had undergone surgical excision of gastric cancer (65 women and 79 men; age range, 3890 years; mean, 68 years). Thirty-nine of the 144 patients had early gastric cancer, and 105 had advanced gastric cancer. Early gastric cancer is limited to the mucosa or the mucosa and submucosa, regardless of nodal status. Advanced gastric cancer is a tumor that has invaded beyond the submucosa. ${ }^{17}$ Primary gastric cancers exhibiting a hepatoid component were regarded as hepatoid adenocarcinomas. Out of 144 primary gastric cancers, 6 cases were histologically classified as hepatoid adenocarcinoma. Out of six hepatoid adenocarcinoma cases, liver metastasis samples were available for two cases. Two patients with liver metastasis were serologically negative for HBV and HCV infection and they did not show any clinical or echographic signs of cirrhosis. Both patients had no history of alcohol abuse. In addition, we used archival formalin-fixed, paraffin-embedded tissues from 52 patients who had undergone surgical excision of hepatocellular carcinoma ( 8 women and 44 men; age range, 44-82 years; mean, 64 years; stage I, 31 cases; stage II, 16 cases; stage III, 5 cases). Normal adult $(n=2,45$ and 57 years) and fetal ( $n=2,10$ and 18 gestational weeks) livers were obtained at autopsy.

\section{Microarray Analysis}

One primary gastric cancer sample (P208T) and corresponding non-neoplastic mucosa were analyzed by genome-wide microarray, as described previously. ${ }^{18}$ Here, we used Affymetrix GeneChip Human Genome U133Plus 2.0 arrays (Affymetrix, Santa Clara, CA, USA). Each transcript on this array is represented by a set of 11 probe pairs, called the probe set. The array contains $>54000$ probe sets, representing 47400 transcripts, including 38500 genes. Five micrograms of total RNA was used to prepare antisense biotinylated RNA with One-cycle Target Labeling and Control Reagent (Affymetrix) as per the manufacturer's instructions. In brief, first-stranded cDNA was synthesized with a T7-RNA polymerase 
promoter-attached oligo(dT) primer followed by second-stranded cDNA synthesis. This cDNA was purified and served as a template in the subsequent in vitro T7-transcription (IVT). The IVT reaction was carried out in the presence of T7 RNA polymerase and biotinylated UTP for cRNA production. The biotinylated cRNAs were then cleaned up and fragmented. The fragmented, biotinylated cRNA was hybridized to the array $\left(45^{\circ} \mathrm{C}\right.$ for $\left.16 \mathrm{~h}\right)$. The procedures for staining, washing, and scanning of arrays were carried out as per the instructions in the Affymetrix technical manual. The expression value (average difference, AD) of each probe was calculated with GeneChip Operating Software Version 1.1 (Affymetrix). The mean of $\mathrm{AD}$ values in each experiment was 1000 to reliably compare variable multiple arrays.

\section{Quantitative RT-PCR}

Total RNA was extracted with an RNeasy Mini Kit (Qiagen, Valencia, CA, USA), and $1 \mu \mathrm{g}$ of total RNA was converted to cDNA with a First Strand cDNA Synthesis Kit (Amersham Biosciences, Piscataway, NJ, USA). Quantitation of PLUNC mRNA levels in human tissue samples was done by real-time fluorescence detection, as described previously. ${ }^{19}$ PLUNC primer sequences were $5^{\prime}$-CAG TTGCCT TCT CTC CGA GG-3' and $5^{\prime}$-CAT GGG ATG TTA CAC ACGCC-3'. PCR was performed with an SYBR Green PCR Core Reagents Kit (Applied Biosystems, Foster City, CA, USA). Real-time detection of the emission intensity of SYBR Green bound to doublestranded DNA was performed with an ABI PRISM 7700 Sequence Detection System (Applied Biosystems), as described previously. ${ }^{20} A C T B$-specific PCR products were amplified from the same RNA samples and served as internal controls.

\section{Western Blotting}

Western blotting was performed as described previously. ${ }^{21}$ Lysates $(40 \mu \mathrm{g})$ were solubilized in Laemmli sample buffer by boiling and then subjected to $12 \%$ SDS-polyacrylamide gel electrophoresis followed by electrotransfer onto a nitrocellulose filter. The filter was incubated with the primary antibody against PLUNC (goat polyclonal, dilution 1:500; R\&D Systems, Abingdon, UK). Peroxidaseconjugated anti-goat IgG was used in the secondary reaction. Immunocomplexes were visualized with an ECL Western Blot Detection System (Amersham Biosciences). $\beta$-Actin (Sigma, St Louis, MO, USA) was also stained as a loading control.

\section{Immunohistochemistry}

Formalin-fixed, paraffin-embedded samples were sectioned, deparaffinized, and stained with H\&E to ensure that the sectioned block contained tumor cells. Adjacent sections were then stained immunohistochemically. Antigen retrieval was done by microwave heating in citrate buffer $(\mathrm{pH}$ 6.0) for 30 min for PLUNC, HepPar1, cytokeratin 19 (CK19), cytokeratin 20 (CK20), and polyclonal carcinoembryonic antigen ( $\mathrm{p}-\mathrm{CEA}$ ). After peroxidase activity was blocked with $3 \% \mathrm{H}_{2} \mathrm{O}_{2}$-methanol for $10 \mathrm{~min}$, sections were incubated with normal goat serum (Dako Cytomation, Carpinteria, CA, USA) for $20 \mathrm{~min}$ to block nonspecific antibody-binding sites. Sections were incubated with the primary antibodies against PLUNC (the same antibody used in western blotting to PLUNC, dilution 1:50), alpha-fetoprotein (AFP) (C3, 1:20; Novocastra, Newcastle, UK), HepPar1 (OCH1E5, 1:20; Dako Cytomation), CK19 (RCK108, 1:50; Dako Cytomation), CK20 (Ks20.8, 1:50; Dako Cytomation), and p-CEA (1:1000; Dako Cytomation) for $1 \mathrm{~h}$ at room temperature, followed by incubations with biotinylated anti-goat, antirabbit, or anti-mouse IgG and peroxidase-labeled streptavidin for $10 \mathrm{~min}$ each. Staining was completed with $10 \mathrm{~min}$ incubation with the substratechromogen solution. The sections were counterstained with $0.1 \%$ hematoxylin. The staining results were recorded in semiquantitative fashion as follows: 0 , absence of staining; $1+$, any tumor cell stained to $10 \%$ of tumor cells stained; $2+, 11-50 \%$ of tumor cells stained; and $3+$, more than $50 \%$ of the tumor cells stained.

\section{Statistical Methods}

Associations between clinicopathologic parameters and PLUNC expression were analyzed by $\chi^{2}$-test. Kaplan-Meier survival curves were constructed for PLUNC-positive and PLUNC-negative patients. Survival rates were compared between PLUNC-positive and PLUNC-negative groups. Differences between survival curves were tested for statistical significance by log-rank test. ${ }^{22} \mathrm{~A} P$-value of less than 0.05 was considered statistically significant.

\section{Results}

\section{Comparison of Gene Expression Profiles from Microarray and SAGE}

The gene expression profiles obtained from the gastric cancer sample (P208T) and corresponding nonneoplastic gastric mucosa sample were compared. To identify ideal biomarkers for gastric cancer, we focused on genes that showed significantly increased expression in gastric cancer. The top 20 genes that showed higher expression in the gastric cancer sample than in the corresponding non-neoplastic gastric mucosa sample by microarray analysis are listed in Table 1. The gene showing the greatest increase in expression in the gastric cancer sample by microarray was PLUNC. Increased expression of the MAGE genes 
Table 1 Comparison of gene expression profiles from microarray and SAGE

\begin{tabular}{|c|c|c|c|c|c|c|}
\hline \multirow{3}{*}{ Symbol } & \multicolumn{2}{|c|}{ Microarray } & \multirow{3}{*}{ Fold } & \multicolumn{3}{|c|}{$S A G E^{\mathrm{a}}$} \\
\hline & \multicolumn{2}{|r|}{ Intensity } & & \multirow[t]{2}{*}{ Symbol } & \multicolumn{2}{|c|}{ Tag counts } \\
\hline & $P 208 T^{\mathrm{b}}$ & Non-neoplastic mucosa & & & $P 208 T^{\mathrm{b}}$ & Normal stomach ${ }^{\mathrm{c}}$ \\
\hline PLUNC & 74421 & 31 & 2401 & TSG101 & 41 & 0 \\
\hline$M A G E A 12$ & 7492 & 8 & 937 & Transcribed locus & 32 & 0 \\
\hline$L A C R T$ & 15873 & 21 & 756 & CTNND1, CYP20A1 & 28 & 0 \\
\hline$M A G E A 6$ & 15966 & 31 & 515 & $B C L 2 L 2$ & 50 & 1 \\
\hline$M A G E A 2$ & 4266 & 12 & 356 & No match & 24 & 0 \\
\hline$M A G E A 3$ & 18963 & 59 & 321 & TUSC3 & 21 & 0 \\
\hline FN1 & 2818 & 9 & 313 & ELOVL5 & 20 & 0 \\
\hline NTRK2 & 2123 & 7 & 303 & S100A9 & 17 & 0 \\
\hline HOXА10 & 4481 & 16 & 280 & KRTHB1, PHYHD1 & 16 & 0 \\
\hline COL11A1 & 2303 & 10 & 230 & $P A W R$ & 16 & 0 \\
\hline FGFR2 & 5593 & 25 & 224 & USP7 & 14 & 0 \\
\hline C20orf186 & 4711 & 21 & 224 & KRT7, SHзBP2 & 14 & 0 \\
\hline$N X F 3$ & 1820 & 10 & 182 & CTSL & 14 & 0 \\
\hline LECT1 & 3348 & 24 & 140 & SEC11L1, WIF1 & 26 & 1 \\
\hline SPP1 & 288 & 2 & 144 & No match & 12 & 0 \\
\hline SH3BP4 & 1214 & 10 & 121 & TFF3 & 12 & 0 \\
\hline$H M 74$ & 22034 & 209 & 105 & $T K T$ & 12 & 0 \\
\hline FLJ20300 & 9617 & 96 & 100 & DNAJC10 & 12 & 0 \\
\hline LOC 284527 & 676 & 7 & 97 & No match & 11 & 0 \\
\hline SLC19A3 & 1631 & 17 & 96 & DDOST & 11 & 0 \\
\hline
\end{tabular}

${ }^{\mathrm{a}}$ The 20 most upregulated genes in gastric cancer (P208T) compared with normal stomach by SAGE analysis was determined previously. ${ }^{8}$

${ }^{\mathrm{b}}$ Gastric cancer sample (60-year-old man, T4N3M0, stage IV, poorly differentiated adenocarcinoma).

${ }^{\mathrm{c}}$ SAGE data from normal gastric epithelia (GSM784, SAGE normal gastric body epithelial, El-Rifai et al ${ }^{23}$ ).

(MAGEA2, MAGEA3, MAGEA6, and MAGEA12), ${ }^{24}$ FN1 (encoding fibronectin 1), ${ }^{25}$ and FGFR2 (encoding fibroblast growth factor 2$)^{26}$ has been reported previously. The 20 genes with the greatest increase in expression in gastric cancer compared with normal stomach $^{23}$ by SAGE analysis are shown in Table 1. Surprisingly, the 20 most upregulated genes identified by microarray were quite different from those identified by SAGE, indicating that genes upregulated in gastric cancer are not always detected by SAGE. We reviewed the expression level of PLUNC with our SAGE data. The sequence of the SAGE tag that represents PLUNC was TGCCTCACCT, and this sequence appeared only three times in the P208T SAGE data. Because expression of PLUNC has not been investigated in gastric cancer, we decided to analyze PLUNC expression in gastric cancer. Quantitative RT-PCR was performed to investigate the specificity of PLUNC expression. As shown in Figure 1a, PLUNC expression was clearly detected in adult trachea and to a lesser extent in adult lung. Expression of PLUNC was not detected in any other normal organs, including stomach. These results are consistent with those of a previous report. ${ }^{13}$ PLUNC was expressed in P208T, whereas other gastric cancer tissue samples did not express PLUNC.

\section{Immunohistochemical Analysis of PLUNC in Gastric Cancer}

We observed upregulation of PLUNC mRNA in gastric cancer tissue; however, the expression pattern of PLUNC protein in gastric cancer remains unclear. To address this issue, we performed immunostaining of PLUNC. We first tested the specificity of the anti-PLUNC antibody. Western blotting of lysates from normal adult trachea and normal stomach was performed. The anti-PLUNC antibody detected an approximately $27 \mathrm{kDa}$ band in adult trachea (Figure 1b). These results are consistent with our quantitative RT-PCR data for PLUNC. Immunostaining of P208T revealed that PLUNC was present in cytoplasm of tumor cells but not in corresponding non-neoplastic mucosa (Figure 1c). Stromal cells showed weak or no staining of PLUNC. Staining of PLUNC was not observed in the remaining three gastric cancer samples used for quantitative RT-PCR. Taken together, these data show that this anti-PLUNC antibody specifically recognizes PLUNC protein.

We next performed immunohistochemical analysis of PLUNC in 140 human gastric cancer tissue samples. Strong cytoplasmic staining of PLUNC was found in 12 (9\%) of 140 gastric cancer samples. Only tumor cells were positive for PLUNC. Stromal cells were not stained. Corresponding non-neoplastic gastric mucosa, including intestinal metaplasia, was not stained. In gastric cancer tissue, staining of PLUNC was heterogeneous, and among 12 PLUNC-positive gastric cancer cases, less than $1 \%$ of tumor cells were stained in 9 gastric cancer cases. In the remaining three gastric cancer cases, more than $30 \%$ of tumor cells were stained. We then analyzed the relation of PLUNC expression to 

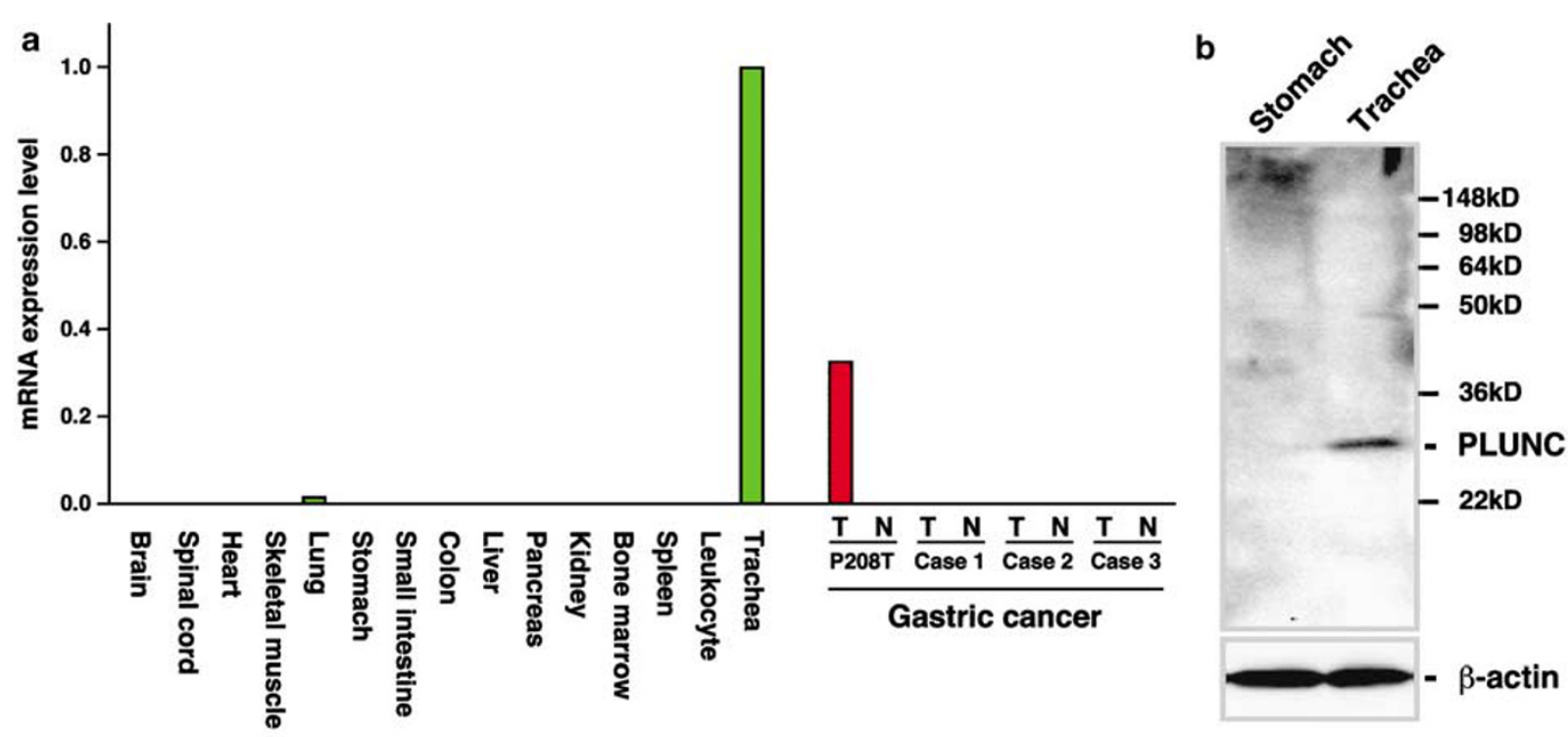

C
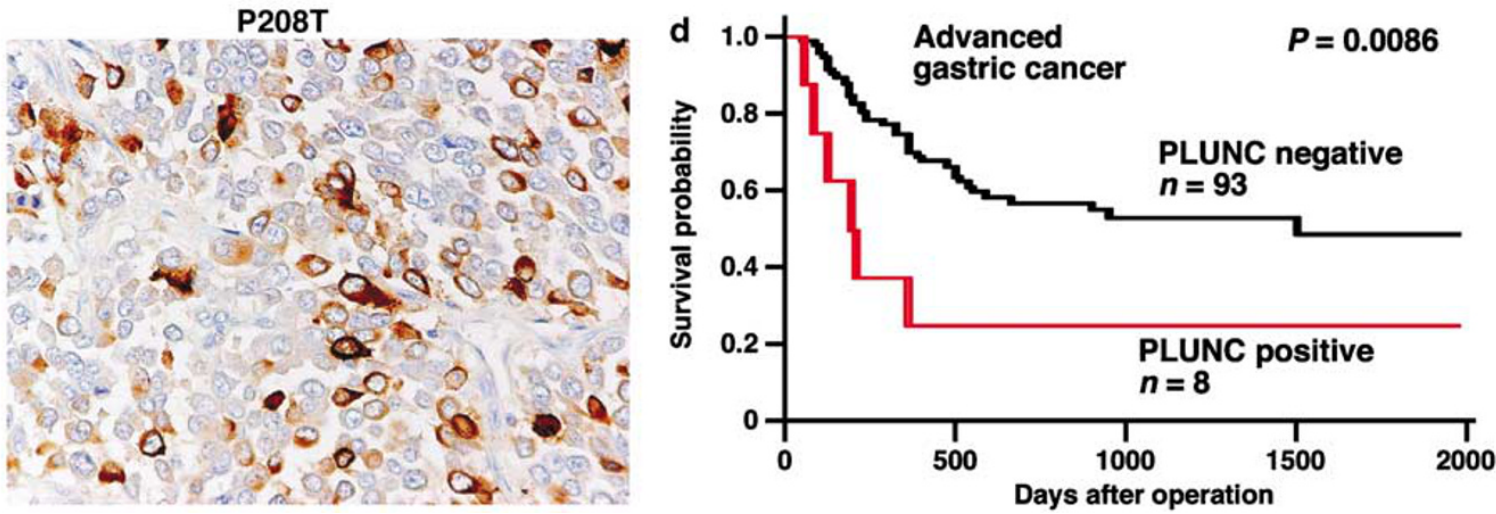

Figure 1 (a) Quantitative RT-PCR analysis of PLUNC in 15 normal tissues, four gastric cancer cases (T), and corresponding nonneoplastic mucosa (N). Case P208T was analyzed by SAGE and microarray. The units are arbitrary, and we calculated the level of PLUNC mRNA expression by standardization to $1.0 \mu \mathrm{g}$ of total RNA from trachea as 1.0. (b) Western blot analysis of PLUNC with anti-PLUNC antibody. An approximately $27 \mathrm{kDa}$ band is present in lysate of normal trachea. (c) Immunostaining of PLUNC in gastric cancer (Case P208T). Focal-positive staining for PLUNC in the cytoplasm is observed. (d) Prognostic value of PLUNC staining. Patients with gastric cancer showing PLUNC expression had a significantly worse survival rate than patients without PLUNC expression $(P=0.0086$, log-rank test).

clinicopathologic characteristics. No correlation was found between PLUNC expression and depth of invasion, lymph node metastasis, or tumor stage (Table 2). In contrast, among 101 advanced gastric cancer cases followed up at the hospital, patients with PLUNC-positive gastric cancer had a significantly worse survival rate than those patients with PLUNC-negative gastric cancer $(P=0.0086$, log-rank test, Figure 1d). Interestingly, of the three gastric cancer cases with more than $30 \%$ of tumor cells stained, two cases showed hepatoid adenocarcinoma of the stomach. The remaining one case was $\mathrm{P} 208 \mathrm{~T}$ and we confirmed that P208T was not gastric hepatoid adenocarcinoma. It has been reported that gastric hepatoid adenocarcinoma shows canalicular staining pattern of $\mathrm{p}$-CEA,${ }^{27}$ and we confirmed that P208T displayed cytoplasmic pattern of p-CEA (data not shown). PLUNC staining was found more frequently in gastric hepatoid adenocarcinoma $(2 / 2$, $100 \%$ ) than in other types (well and poorly differentiated adenocarcinoma) of gastric cancer (10/138, $7 \%, P=0.0007, \chi^{2}$-test).

\section{Immunohistochemical Analysis of PLUNC in Hepatoid} Adenocarcinoma of the Stomach

It is well known that gastric hepatoid adenocarcinoma has an aggressive clinical course and poor survival. ${ }^{28,29}$ Its biologic behavior is mainly due to its extensive hematogenous metastasis to the liver and early and frequent involvement of the lymph nodes. Gastric hepatoid adenocarcinoma closely mimics and is even indistinguishable from hepatocellular carcinoma. This makes differential diagnosis challenging, especially when the primary tumor is unknown and the first diagnosis has to be established by liver biopsy. Therefore, there is an urgent need for specific biomarkers of hepatoid adenocarcinoma. We performed immunohistochemical analysis 
Table 2 Association of PLUNC expression with clinicopathologic features of gastric cancer

\begin{tabular}{|c|c|c|c|}
\hline & \multicolumn{2}{|c|}{ PLUNC expression (\%) } & \multirow[t]{2}{*}{$\mathrm{P}$-value } \\
\hline & Positive & Negative & \\
\hline \multicolumn{4}{|l|}{$T$ grade } \\
\hline T1 & $4(10)$ & 35 & 0.9157 \\
\hline $\mathrm{T} 2 / \mathrm{T} 3 / \mathrm{T} 4$ & $8(8)$ & 93 & \\
\hline \multicolumn{4}{|l|}{$N$ grade } \\
\hline No & $5(7)$ & 62 & 0.8833 \\
\hline N1/N2/N3 & $7(10)$ & 66 & \\
\hline \multicolumn{4}{|l|}{$M$ grade } \\
\hline Mo & $11(8)$ & 120 & 0.7784 \\
\hline M1 & $1(11)$ & 8 & \\
\hline \multicolumn{4}{|l|}{ Stage } \\
\hline Stage I & $4(7)$ & 56 & 0.6394 \\
\hline Stage II & $3(12)$ & 22 & \\
\hline Stage III & $2(8)$ & 23 & \\
\hline Stage IV & $3(10)$ & 27 & \\
\hline \multicolumn{4}{|l|}{ Histologic type } \\
\hline $\begin{array}{l}\text { Well-differentiated } \\
\text { adenocarcinoma }\end{array}$ & $6(7)$ & 75 & $0.0007^{\mathrm{b}}$ \\
\hline $\begin{array}{l}\text { Poorly differentiated } \\
\text { adenocarcinoma }\end{array}$ & $4(7)$ & 53 & \\
\hline Hepatoid adenocarcinoma & $2(100)$ & 0 & \\
\hline
\end{tabular}

${ }^{\mathrm{a}} \chi^{2}$-Test.

${ }^{\mathrm{b}}$ Well-differentiated and poorly differentiated adenocarcinoma vs hepatoid adenocarcinoma.

of PLUNC in four additional cases of hepatoid adenocarcinoma to investigate the potential utility of PLUNC immunostaining in the diagnosis of hepatoid adenocarcinoma. Among six cases of hepatoid adenocarcinoma (two cases from immunohistochemical analysis in 140 gastric cancer cases plus four additional cases), staining of PLUNC was observed in all six primary tumors. It has been reported that most hepatoid adenocarcinoma cases contain coexistent tubular or papillary adenocarcinoma components within the tumor. ${ }^{30}$ All six gastric hepatoid adenocarcinoma cases contained both tubular/papillary adenocarcinoma and hepatoid adenocarcinoma components. out of the six hepatoid adenocarcinoma cases, PLUNC staining was observed in both the hepatoid adenocarcinoma component (Figure 2a and b) and the tubular/ papillary adenocarcinoma component (Figure 2c and d). We confirmed that hepatoid adenocarcinoma component showed a canalicular pattern of p-CEA staining (Figure 2a, inset). The PLUNC staining was preferentially found in the tubular/papillary adenocarcinoma component of the primary tumors. In some cases, even when PLUNC-positive tumor cells were found in the tubular/papillary adenocarcinoma component near the hepatoid adenocarcinoma component, PLUNC was not stained in the hepatoid adenocarcinoma component (Figure 2e).Out of the six cases of hepatoid adenocarcinoma, the hepatoid adenocarcinoma component contained 5-10\% PLUNC-positive tumor cells, whereas tubular/papillary adenocarcinoma component contained $\leq 70 \%$ PLUNC-positive tumor cells (Table 3). Out of the six cases of hepatoid adenocarcinoma, liver metastases from two cases were available for immunohistochemistry and were composed of hepatoid adenocarcinoma component. PLUNC staining was observed in both liver metastases (Figure 2f). One case showed 5\% PLUNC-positive tumor cells, and another case contained 20\% PLUNC-positive tumor cells within the metastatic tumor. Adjacent nonneoplastic liver tissues were not stained for PLUNC (Figure 2f). We also performed immunohistochemical analysis of PLUNC in 52 cases of hepatocellular carcinoma, 2 cases of normal adult liver, and 2 cases of fetal liver; however, staining of PLUNC was not observed (data not shown). These results indicate that PLUNC is a good marker to distinguish gastric hepatoid adenocarcinoma from primary hepatocellular carcinoma.

\section{Immunohistochemical Analysis of AFP, HepPar1, CK19, and CK20 in Hepatoid Adenocarcinoma of the Stomach}

Because production of AFP is usually observed in gastric hepatoid adenocarcinoma, we performed immunostaining of AFP. The results are summarized in Table 3. Although all six hepatoid adenocarcinoma cases were positive for AFP, AFP-positive tumor cells were not positive for PLUNC. AFP-positive tumor cells were observed in hepatoid adenocarcinoma components, whereas PLUNC-positive tumor cells were found mainly in tubular/papillary adenocarcinoma components (Figure 3a and b). Even when PLUNC-positive tumor cells were found in hepatoid adenocarcinoma components, PLUNC-positive tumor cells did not express AFP (Figure 3c and d).

It was previously reported that immunohistochemical analyses of HepPar1, CK19, or CK20 are useful to distinguish gastric hepatoid adenocarcinoma from primary hepatocellular carcinoma. ${ }^{27}$ HepPar1 staining is detected more frequently in hepatocellular carcinoma than in gastric hepatoid adenocarcinoma. ${ }^{27}$ Staining for CK19 and CK20 is detected more frequently in gastric hepatoid adenocarcinoma than in hepatocellular carcinoma. ${ }^{27}$ Therefore, we also performed immunostaining of HepPar1, CK19, and CK20 in gastric hepatoid adenocarcinoma and hepatocellular carcinoma (Table 3). The overall results are summarized in Table 4 . Out of six cases of gastric hepatoid adenocarcinoma, four cases showed focal positivity for HepPar1, and all four hepatoid adenocarcinoma cases showed less than 10\% HepPar1-positive tumor cells. The remaining two hepatoid adenocarcinoma cases did not express HepPar1. Staining of HepPar1 was also detected in both liver metastases of hepatoid 

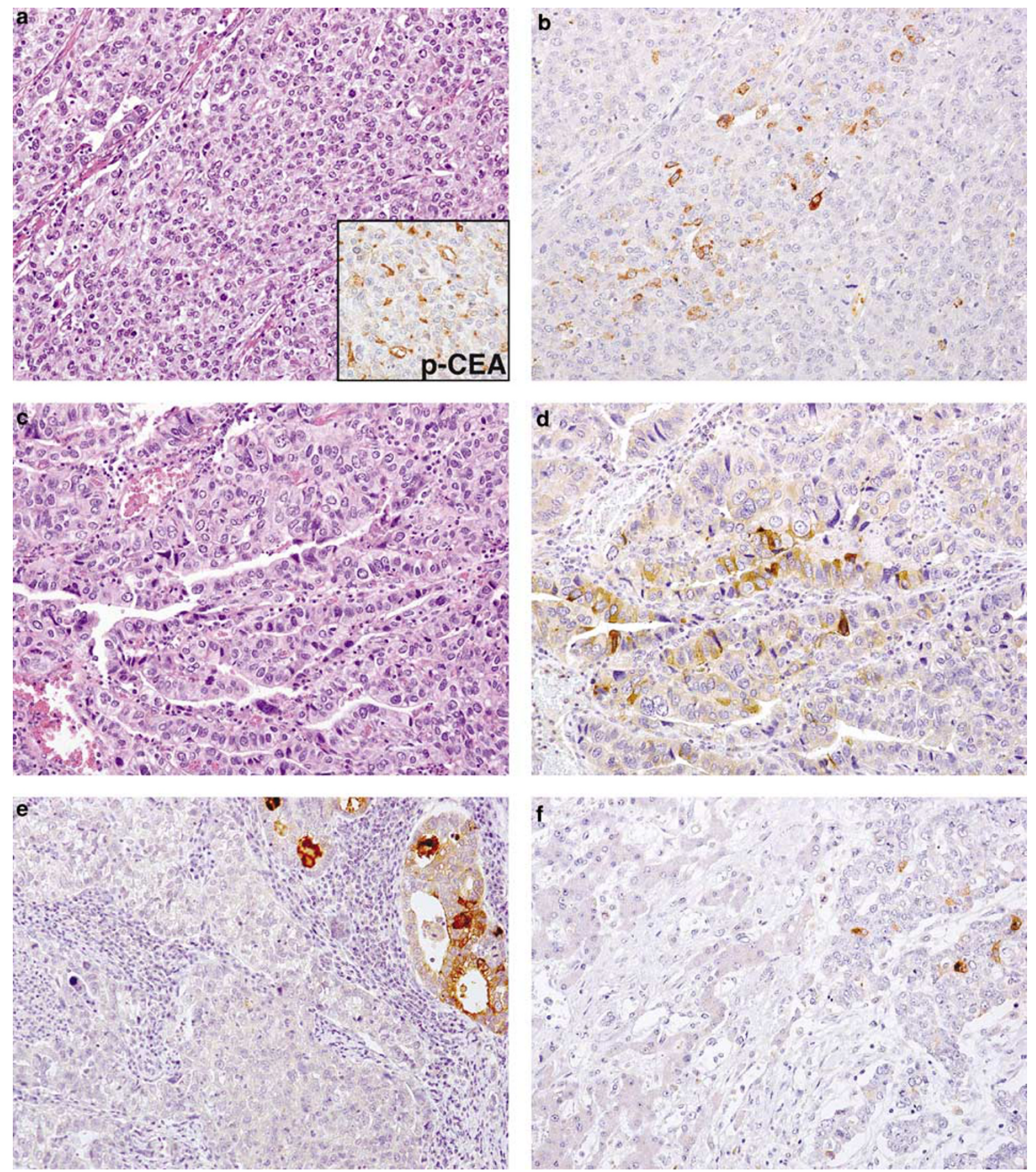

Figure 2 Immunostaining of PLUNC in hepatoid adenocarcinoma of the stomach. (a) H\&E staining of hepatoid adenocarcinoma component of primary tumor. Tumor cells display a solid structure resembling that of hepatocellular carcinoma. Inset: hepatoid adenocarcinoma component showed a canalicular pattern. (b) Corresponding PLUNC staining. Focal-positive staining for PLUNC is observed. (c) H\&E stain of tubular/papillary adenocarcinoma component of primary tumor that consists of polygonal tumor cells with large central nuclei. (d) Corresponding PLUNC staining. Focal-positive staining for PLUNC is observed. (e) The tubular adenocarcinoma component is stained by anti-PLUNC antibody, with luminal and cytoplasmic patterns, whereas the hepatoid adenocarcinoma component shows no staining. (f) Liver metastasis of gastric hepatoid adenocarcinoma. Focal-positive staining for PLUNC is detected, whereas adjacent non-neoplastic hepatocytes are not stained.

adenocarcinoma (Figure 4b). The two liver metastases of hepatoid adenocarcinoma contained less than 10\% HepPar1-positive tumor cells. In contrast, most of the primary hepatocellular carcinoma cases
(45 out of 52 cases, $87 \%$ ) displayed strong and extensive staining $(2+, 10$ cases; $3+, 35$ cases $)$ of HepPar1 (Figure 4c). All six hepatoid adenocarcinoma cases showed CK19 staining (Figure 4d), and 
Table 3 PLUNC, AFP, HepPar1, CK19, and CK20 immunoreactivity in hepatoid adenocarcinoma of the stomach

\begin{tabular}{|c|c|c|c|c|c|c|c|c|c|c|c|}
\hline \multirow[t]{2}{*}{ Case no. } & \multirow[t]{2}{*}{ Organ } & \multicolumn{5}{|c|}{ Hepatoid adenocarcinoma component } & \multicolumn{5}{|c|}{ Tubular/papillary adenocarcinoma component } \\
\hline & & $P L U N C$ & $A F P$ & HepPar1 & CK19 & CK2O & $P L U N C$ & $A F P$ & HepPar1 & CK19 & CK20 \\
\hline 52 & Stomach & $1+$ & $2+$ & $1+$ & $2+$ & 0 & $3+$ & 0 & $1+$ & $2+$ & 0 \\
\hline 126 & Stomach & $1+$ & $1+$ & $1+$ & $2+$ & 0 & $3+$ & 0 & 0 & $3+$ & 0 \\
\hline 141 & Stomach & $1+$ & $1+$ & 0 & $1+$ & $1+$ & $1+$ & 0 & 0 & $1+$ & 0 \\
\hline 142 & Stomach & $1+$ & $1+$ & 0 & $2+$ & 0 & $2+$ & 0 & 0 & $2+$ & $1+$ \\
\hline \multirow[t]{2}{*}{143} & Stomach $^{\mathrm{a}}$ & $1+$ & $2+$ & $1+$ & $1+$ & 0 & 0 & 0 & $1+$ & $1+$ & 0 \\
\hline & Liver & $1+$ & $2+$ & $1+$ & $2+$ & 0 & $-^{\mathrm{b}}$ & - & - & - & - \\
\hline \multirow[t]{2}{*}{144} & Stomach $^{\mathrm{a}}$ & $1+$ & $2+$ & $1+$ & $2+$ & $1+$ & $1+$ & 0 & 0 & $3+$ & 0 \\
\hline & Liver & $2+$ & $3+$ & $1+$ & $3+$ & $1+$ & - & - & - & - & - \\
\hline
\end{tabular}

0 indicates negative; $1+, \leq 10 \% ; 2+, 11-50 \% ; 3+,>50 \%$.

${ }^{\text {a}}$ Primary tumor.

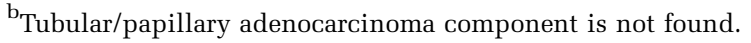

CK19 staining was observed in both liver metastases (Figure 4e). One case showed $80 \%$ CK19-positive tumor cells, and another case contained 20\% CK19positive tumor cells within the metastatic tumor. Forty-four out of 52 (85\%) hepatocellular carcinoma cases showed no staining of CK19, and CK19 staining was observed in eight hepatocellular carcinoma cases ( $1+, 2$ cases; $2+, 3$ cases; $3+, 3$ cases) (Figure 4f). Two out of six gastric hepatoid adenocarcinoma cases showed focal positivity for CK20, and both hepatoid adenocarcinoma cases showed less than 10\% CK20-positive tumor cells (Figure 4g). CK20 staining was observed in one case of liver metastasis (Figure 4h). Forty-two out of 52 (81\%) hepatocellular carcinoma cases showed no staining of CK20, and CK20 staining was observed in 10 hepatocellular carcinoma cases $(1+, 3$ cases; $2+, 4$ cases; $3+, 3$ cases) (Figure 4i).

\section{Discussion}

Gastric hepatoid adenocarcinoma frequently shows histologic features that mimic hepatocellular carcinoma. It can be very challenging to differentiate hepatoid adenocarcinoma from hepatocellular carcinoma on the basis of morphology alone, especially when a specimen is limited, such as small tissue biopsy specimens. Immunoreactivity for AFP, alpha1 antitrypsin, ${ }^{30}$ and Glypican $3^{31}$ in hepatoid adenocarcinoma has been reported. Moreover, albumin mRNA has also been detected in hepatoid adenocarcinoma by in situ hybridization. ${ }^{32}$ These molecules are expressed in normal adult or fetal liver and show various degrees of utility in distinguishing gastric hepatoid adenocarcinoma from conventional gastric cancer; however, these molecules are also expressed in hepatocellular carcinoma. Therefore, there is no reliable biomarker to distinguish hepatoid adenocarcinoma from hepatocellular carcinoma. In this study, we observed overexpression of PLUNC in gastric cancer with two comprehensive gene expression profiling methods, SAGE and microarray. Although only $7 \%$ of conventional gastric cancer cases showed focal immunostaining of PLUNC, extensive staining of PLUNC was observed in all six cases of hepatoid adenocarcinoma. Hepatocellular carcinoma cases did not show staining of PLUNC, whereas both cases of liver metastases of hepatoid adenocarcinoma were positive for PLUNC. These results indicate that PLUNC is a good marker to distinguish hepatoid adenocarcinoma from hepatocellular carcinoma. In this study, staining of PLUNC was not detected in normal adult and fetal liver. In gastric hepatoid adenocarcinoma, PLUNC-positive tumor cells were not stained by AFP. These findings led us to speculate that PLUNC is not involved in hepatocyte differentiation and that this is the reason why immunostaining of PLUNC can distinguish gastric hepatoid adenocarcinoma from hepatocellular carcinoma.

What are the unique features and utility of PLUNC immunostaining? It should be mentioned here that all the samples analyzed by immunostaining in the present study were surgically resected specimens. For surgical resection of a tumor, pathologic diagnosis from a biopsy specimen is essential. Because this is the first investigation of PLUNC in gastric cancer, we analyzed surgically resected specimens to investigate the precise distributions of PLUNCpositive cells. In this study, PLUNC was preferentially expressed in the tubular/papillary adenocarcinoma components of primary tumors. In gastric hepatoid adenocarcinoma, the tubular/papillary adenocarcinoma component tends to be located superficially in the primary tumor, whereas the hepatoid adenocarcinoma component tends to be located in the deeper parts of the primary tumor. ${ }^{27,33}$ In fact, PLUNC-positive tumor cells were frequently found in superficial areas of gastric hepatoid adenocarcinoma. Therefore, PLUNC immunostaining can be used to test gastric biopsy specimens because only superficial areas are obtained by gastric biopsy. Furthermore, among 138 conventional gastric cancer cases, less than $1 \%$ of tumor cells were stained in nine gastric cancer cases, and only one gastric cancer case (P208T) showed 


\section{PLUNC}
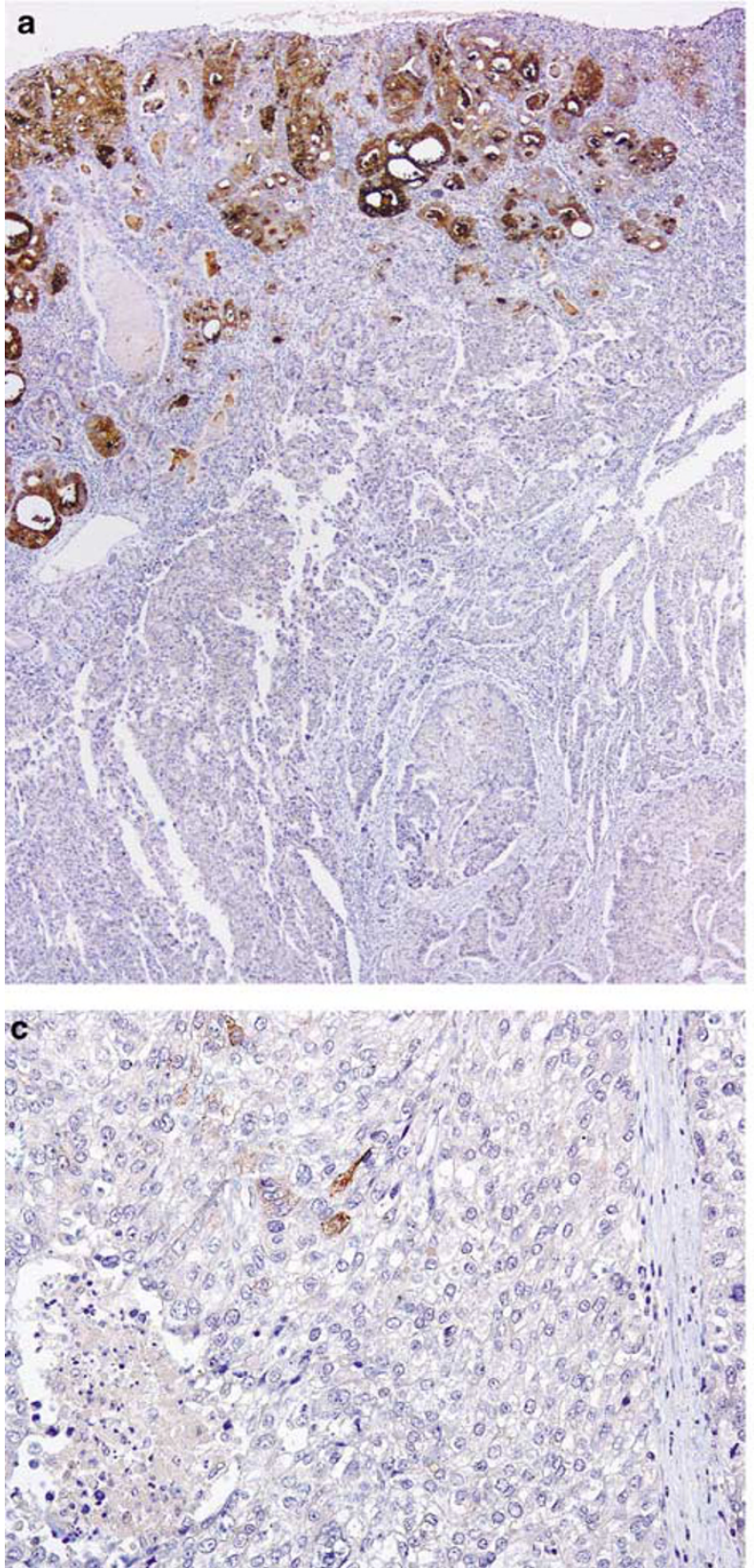
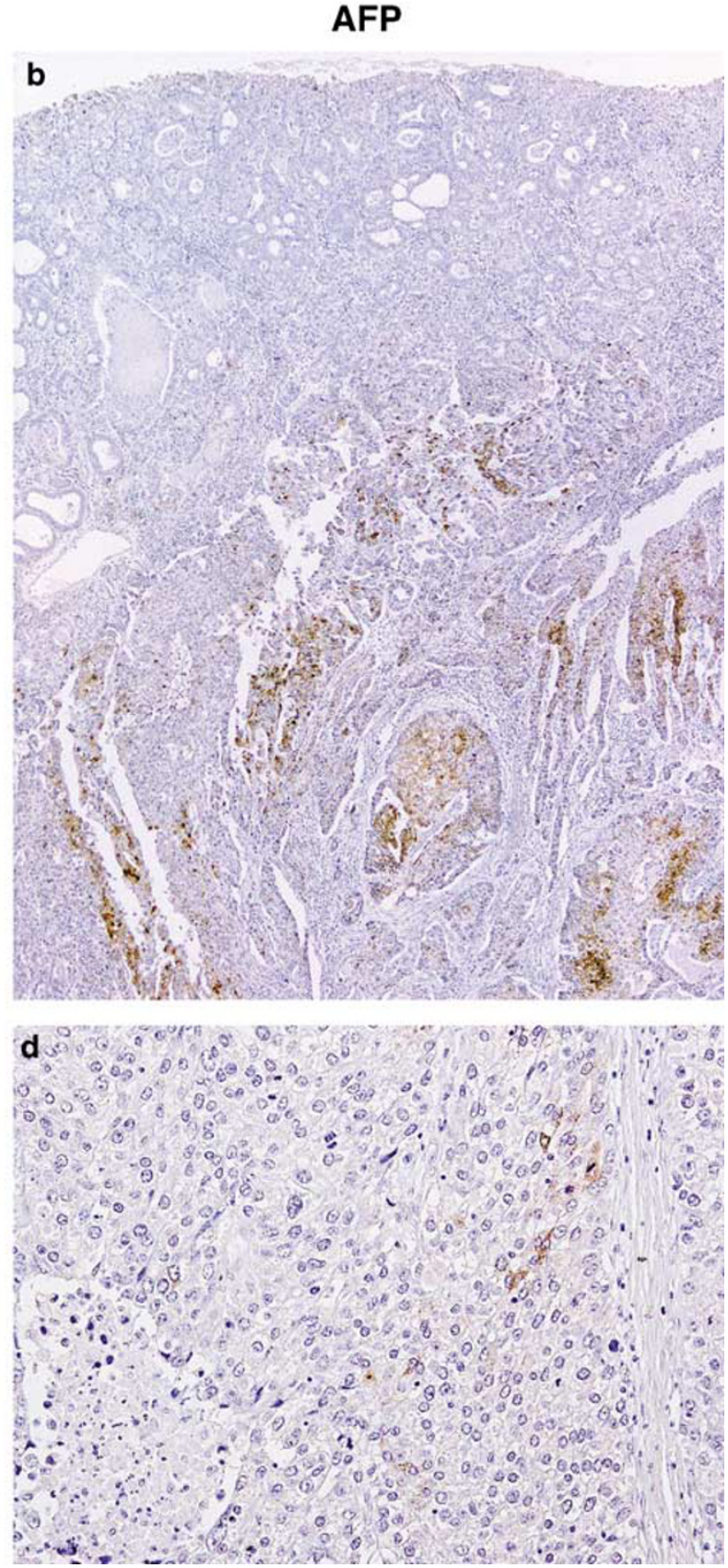

Figure 3 Immunostaining of PLUNC and AFP in hepatoid adenocarcinoma of the stomach. PLUNC staining is observed in the tubular adenocarcinoma component (a), whereas AFP staining is found in the hepatoid adenocarcinoma component (b). In the hepatoid adenocarcinoma component, PLUNC-positive tumor cells (c) are negative for AFP (d).

extensive staining of PLUNC. These results indicate that immunostaining of PLUNC can differentiate gastric hepatoid adenocarcinoma from conventional gastric cancer. Because PLUNC is frequently stained in the tubular/papillary adenocarcinoma component of gastric hepatoid adenocarcinoma, PLUNC staining can assist in diagnosing gastric hepatoid adenocarcinoma even when the hepatoid adenocarcinoma component is not found in gastric biopsy specimens.

The liver is the most common organ for metastasis by gastric hepatoid adenocarcinoma. It is difficult in some cases to distinguish a liver metastasis of hepatoid adenocarcinoma from a primary hepatocellular carcinoma with liver biopsy specimens because gastric hepatoid adenocarcinoma frequently shows histologic and immunostaining features that mimic those of hepatocellular carcinoma. It was previously reported that immunostaining for HepPar1, CK19, or CK20 is useful to distinguish gastric hepatoid adenocarcinoma from primary hepatocellular carcinoma. ${ }^{27}$ In fact, most primary hepatocellular carcinoma showed extensive staining of HepPar1, whereas only focal staining of HepPar1 was observed in gastric hepatoid adenocarcinoma in 
Table 4 Summary of PLUNC, AFP, HepPar1, CK19, and CK20 immunostaining

\begin{tabular}{lccc}
\hline & & No. of positive ${ }^{\mathrm{a}}$ cases \\
\cline { 2 - 4 } & $\begin{array}{c}\text { Primary gastric hepatoid } \\
\text { adenocarcinoma (n=6) }\end{array}$ & $\begin{array}{c}\text { Liver metastasis of gastric } \\
\text { hepatoid adenocarcinoma (n=2) }\end{array}$ & $\begin{array}{c}\text { Hepatocellular } \\
\text { carcinoma (n=52) }\end{array}$ \\
\hline PLUNC & $6(100 \%)$ & $2(100 \%)$ & $0(0 \%)$ \\
AFP & $6(100 \%)$ & $2(100 \%)$ & $11(21 \%)$ \\
HepPar1 & $4(67 \%)$ & $2(100 \%)$ & $48(92 \%)$ \\
CK19 & $6(100 \%)$ & $2(100 \%)$ & $8(15 \%)$ \\
CK20 & $2(33 \%)$ & $1(50 \%)$ & $10(19 \%)$ \\
\hline
\end{tabular}

${ }^{\mathrm{a}} 1+, 2+$, and $3+$ cases were considered positive.

Primary gastric hepatoid adenocarcinoma
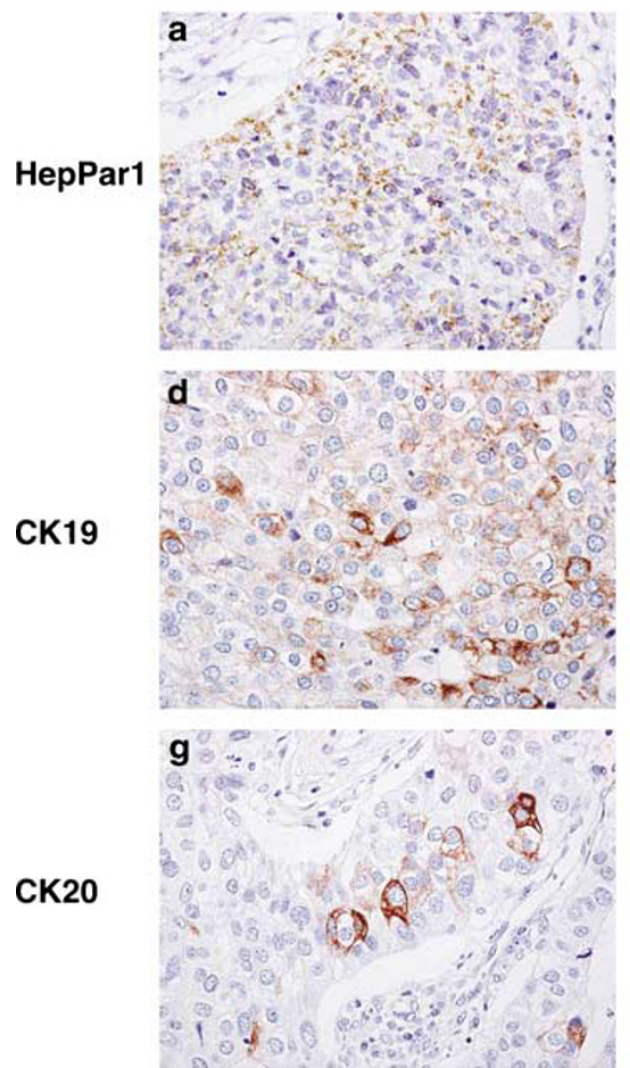

\section{Liver metastasis of gastric hepatoid adenocarcinoma}
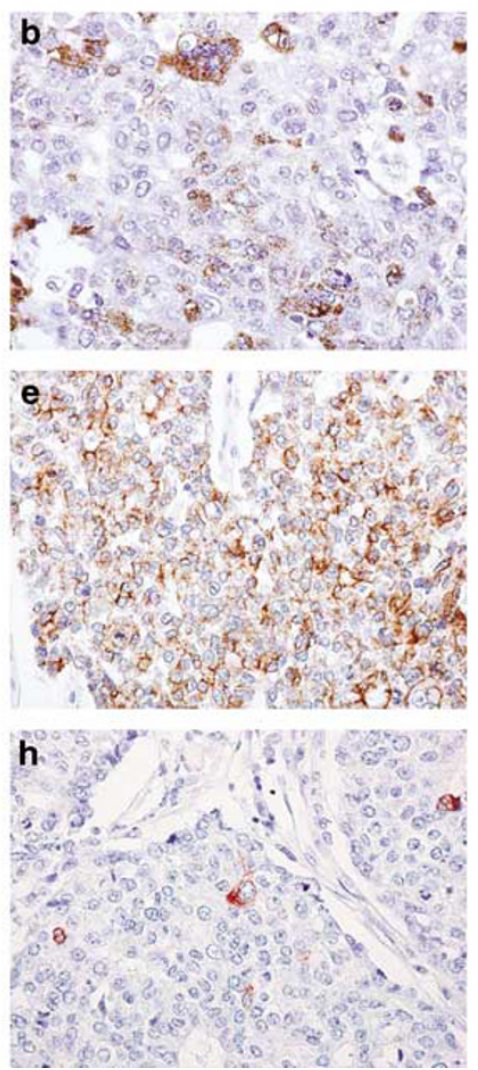

Hepatocellular carcinoma
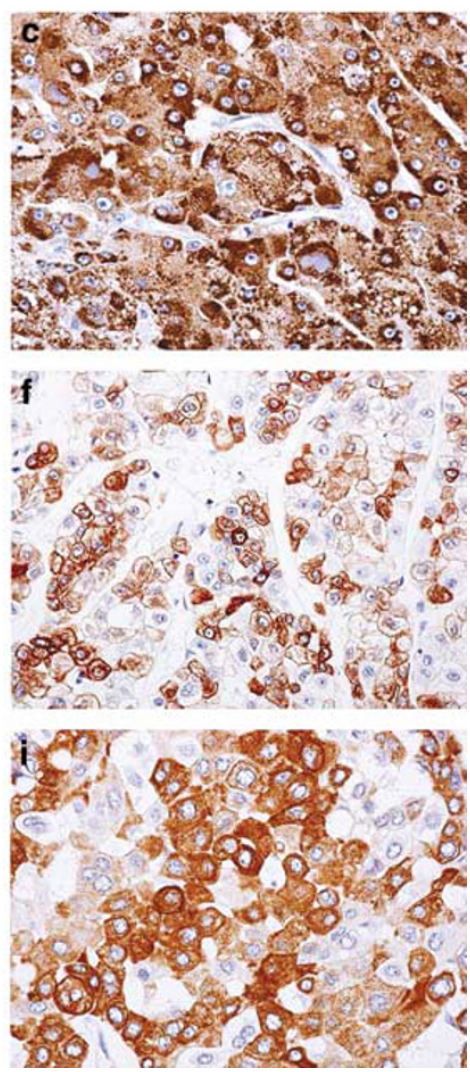

Figure 4 Immunostaining of HepPar1 (a-c), CK19 (d-f), and CK20 (g-i) in primary gastric hepatoid adenocarcinoma, liver metastasis of gastric hepatoid adenocarcinoma, and hepatocellular carcinoma. (a) In gastric hepatoid adenocarcinoma, hepatoid adenocarcinoma component shows focal-positive for HepPar1 within the primary tumor. (b) In liver metastasis of gastric hepatoid adenocarcinoma, focal-positive staining for HepPar1 is found. (c) Hepatocellular carcinoma cases show strong and extensive staining for HepPar1. (d) Cytoplasmic staining of CK19 is detected in hepatoid adenocarcinoma component in primary gastric hepatoid adenocarcinoma. (e) Diffuse CK19 staining is observed in liver metastasis of gastric hepatoid adenocarcinoma. (f) Some hepatocellular carcinoma cases show focal CK19 staining. (g) Focal CK20 staining is found in primary gastric hepatoid adenocarcinoma. (h) In liver metastasis of gastric hepatoid adenocarcinoma, focal-positive staining for CK20 is detected. (i) Some hepatocellular carcinoma cases show focal CK20 staining.

this study. Staining for CK19 and CK20 was detected more frequently in gastric hepatoid adenocarcinoma than in hepatocellular carcinoma in the present study. Because only six gastric hepatoid adenocarcinoma cases and two liver metastases of hepatoid adenocarcinoma were investigated in this study, it is difficult to conclude which is the better marker.
At least however, because PLUNC staining was found only in gastric hepatoid adenocarcinoma, but not in hepatocellular carcinoma, PLUNC is a specific marker to distinguish hepatoid adenocarcinoma from hepatocellular carcinoma.

In this study, the 20 genes showing the greatest increase in expression on microarray were quite 
different from those obtained by SAGE. Investigation of the difference between microarray and SAGE is beyond the scope of this study and will be described elsewhere.

In summary, we found that PLUNC is expressed in gastric hepatoid adenocarcinoma but not primary hepatocellular carcinoma. Although larger trials are required, this initial study shows the potential of PLUNC immunostaining to serve as a marker to distinguish metastatic hepatoid adenocarcinoma from primary hepatocellular carcinoma. In conventional gastric cancer cases, patients with PLUNCpositive gastric cancer had a significantly worse survival rate than those patients with PLUNCnegative gastric cancer; therefore, expression of PLUNC may be a key factor mediating the malignant behavior of gastric hepatoid adenocarcinoma.

\section{Acknowledgements}

We thank Ms Emiko Hisamoto and Mr Masayuki Ikeda for their excellent technical assistance and advice. This work was carried out with the kind cooperation of the Research Center for Molecular Medicine, Faculty of Medicine, Hiroshima University. We thank the Analysis Center of Life Science, Hiroshima University, for the use of their facilities. This work was supported, in part, by Grants-in-Aid for Cancer Research from the Ministry of Education, Culture, Science, Sports, and Technology of Japan; by the program for promotion of Fundamental Studies in Health Sciences of the National Institute of Biomedical Innovation (NiBio); and by a Grant-inAid for the Third Comprehensive 10-Year Strategy for Cancer Control and for Cancer Research (16-15) from the Ministry of Health, Labour and Welfare of Japan.

\section{References}

1 Ohgaki H, Matsukura N. Stomach cancer. In: Stewart BW, Kleihues P (eds). World Cancer Report. IARC Press: Lyon, 2003, pp 197.

2 Yasui W, Oue N, Kitadai Y, et al. Recent advances in molecular pathobiology of gastric carcinoma. In: Kaminishi M, Takubo K, Mafune K (eds). The Diversity of Gastric Carcinoma Pathogenesis: Diagnosis, and Therapy. Springer: Tokyo, 2005, pp 51-71.

3 Kondo T, Oue N, Mitani Y, et al. Loss of heterozygosity and histone hypoacetylation of the PINX1 gene are associated with reduced expression in gastric carcinoma. Oncogene 2005;24:157-164.

4 Kurayoshi M, Oue N, Yamamoto $\mathrm{H}$, et al. Expression of Wnt-5a is correlated with aggressiveness of gastric cancer by stimulating cell migration and invasion. Cancer Res 2006;66:10439-10448.

5 Yasui W, Oue N, Ito R, et al. Search for new biomarkers of gastric cancer through serial analysis of gene expression and its clinical implications. Cancer Sci 2004;95:385-392.
6 Lockhart DJ, Dong H, Byrne MC, et al. Expression monitoring by hybridization to high-density oligonucleotide arrays. Nat Biotechnol 1996;14:1675-1680.

7 Velculescu VE, Zhang L, Vogelstein B, et al. Serial analysis of gene expression. Science 1995;270: 484-487.

8 Oue N, Hamai Y, Mitani Y, et al. Gene expression profile of gastric carcinoma: identification of genes and tags potentially involved in invasion, metastasis, and carcinogenesis by serial analysis of gene expression. Cancer Res 2004;64:2397-2405.

9 Aung PP, Oue N, Mitani Y, et al. Systematic search for gastric cancer-specific genes based on SAGE data: melanoma inhibitory activity and matrix metalloproteinase-10 are novel prognostic factors in patients with gastric cancer. Oncogene 2006;25:2546-2557.

10 Ishii $\mathrm{M}$, Hashimoto $\mathrm{S}$, Tsutsumi $\mathrm{S}$, et al. Direct comparison of GeneChip and SAGE on the quantitative accuracy in transcript profiling analysis. Genomics 2000;68:136-143.

11 Oue N, Aung PP, Mitani Y, et al. Genes involved in invasion and metastasis of gastric cancer identified by array-based hybridization and serial analysis of gene expression. Oncology 2005;69(Suppl 1):17-22.

12 Weston WM, LeClair EE, Trzyna W, et al. Differential display identification of plunc, a novel gene expressed in embryonic palate, nasal epithelium, and adult lung. J Biol Chem 1999;274:13698-13703.

13 Bingle CD, Bingle L. Characterisation of the human plunc gene, a gene product with an upper airways and nasopharyngeal restricted expression pattern. Biochim Biophys Acta 2000;1493:363-367.

14 Iwao K, Watanabe T, Fujiwara Y, et al. Isolation of a novel human lung-specific gene, LUNX, a potential molecular marker for detection of micrometastasis in non-small-cell lung cancer. Int J Cancer 2001;91: 433-437.

15 Bingle L, Cross SS, High AS, et al. SPLUNC1 (PLUNC) is expressed in glandular tissues of the respiratory tract and in lung tumours with a glandular phenotype. J Pathol 2005;205:491-497.

16 Sobin LH, Wittekind CH, (eds). TNM Classification of Malignant Tumors, 6th edn. Wiley-Liss Inc: New York, 2002, pp 65-68.

17 Hohenberger P, Gretschel S. Gastric cancer. Lancet 2003;362:305-315.

18 Nishigaki M, Aoyagi K, Danjoh I, et al. Discovery of aberrant expression of R-RAS by cancer-linked DNA hypomethylation in gastric cancer using microarrays. Cancer Res 2005;65:2115-2124.

19 Gibson UE, Heid CA, Williams PM. A novel method for real time quantitative RT-PCR. Genome Res 1996;6:995-1001.

20 Kondo $\mathrm{T}$, Oue N, Yoshida $\mathrm{K}$, et al. Expression of POT1 is associated with tumor stage and telomere length in gastric carcinoma. Cancer Res 2004;64: 523-529.

21 Yasui W, Ayhan A, Kitadai Y, et al. Increased expression of p34cdc2 and its kinase activity in human gastric and colonic carcinomas. Int J Cancer 1993;53: 36-41.

22 Mantel N. Evaluation of survival data and two new rank order statistics arising in its consideration. Cancer Chemother Rep 1966;50:163-170.

23 El-Rifai W, Moskaluk CA, Abdrabbo MK, et al. Gastric cancers overexpress S100A calcium-binding proteins. Cancer Res 2002;62:6823-6826. 
24 Inoue $\mathrm{H}$, Mori M, Honda M, et al. The expression of tumor-rejection antigen 'MAGE' genes in human gastric carcinoma. Gastroenterology 1995;109: 1522-1525.

25 David L, Nesland JM, Holm R, et al. Expression of laminin, collagen IV, fibronectin, and type IV collagenase in gastric carcinoma. An immunohistochemical study of 87 patients. Cancer 1994;73:518-527.

26 Ueki T, Koji T, Tamiya S, et al. Expression of basic fibroblast growth factor and fibroblast growth factor receptor in advanced gastric carcinoma. J Pathol 1995;177:353-361.

27 Terracciano LM, Glatz K, Mhawech P, et al. Hepatoid adenocarcinoma with liver metastasis mimicking hepatocellular carcinoma: an immunohistochemical and molecular study of eight cases. Am J Surg Pathol 2003;27:1302-1312.

28 Chang YC, Nagasue N, Abe S, et al. Alpha fetoprotein producing early gastric cancer with liver metastasis: report of three cases. Gut 1991;32:542-545.
29 Ishikura H, Kishimoto T, Andachi H, et al. Gastrointestinal hepatoid adenocarcinoma: venous permeation and mimicry of hepatocellular carcinoma, a report of four cases. Histopathology 1997;31:47-54.

30 Ishikura H, Kirimoto K, Shamoto M, et al. Hepatoid adenocarcinomas of the stomach. An analysis of seven cases. Cancer 1986;58:119-126.

31 Hishinuma M, Ohashi KI, Yamauchi N, et al. Hepatocellular oncofetal protein, glypican 3 is a sensitive marker for alpha-fetoprotein-producing gastric carcinoma. Histopathology 2006;49:479-486.

32 Supriatna Y, Kishimoto T, Uno T, et al. Evidence for hepatocellular differentiation in alpha-fetoprotein-negative gastric adenocarcinoma with hepatoid morphology: a study with in situ hybridisation for albumin mRNA. Pathology 2005;37:211-215.

33 Kishimoto $\mathrm{T}$, Nagai $\mathrm{Y}$, Kato $\mathrm{K}$, et al. Hepatoid adenocarcinoma: a new clinicopathological entity and the hypotheses on carcinogenesis. Med Electron Microsc 2000;33:57-63. 